Invest. pens. crit. (ISSN 1812-3864)

Vol. 5, No. 2, mayo-agosto 2017

pp. $21-36$

\title{
Los Dibujos Animados: herramienta para la educación'1.
}

\section{Gabriela Mornhinweg ${ }^{2}$, Luis Carlos Herrera M. ${ }^{3}$}

Recibido: 24 de mayo de 2017

Aceptado: 05 de julio de 2017

\begin{abstract}
Over the years, the media has positioned itself as the fourth power in the world to be a transmitting tool of "valuable" content for society. This content is deployed in different genres, with the aim of transmitting messages, imparting concepts and entertaining, using different techniques, especially cartoons, to capture in their audience the childhood, especially. These drawings are produced in a variety of colors, sizes and themes, often presenting content not suitable for your audience. This research proposes, first, a classification of these cartoons, according to their content. And second, understand the cartoon preferences that sixth grade students from urban official elementary schools demonstrate. The research focused on two case studies, the highest and lowest score according to TERCE tests.
\end{abstract}

Keywords: Cartoon; TERCE Tests; Education; Pedagogy; Educational innovation.

\section{Resumen}

A través de los años, los medios de comunicación se han posicionado como el cuarto poder del estado al ser una herramienta transmisora de contenido "valioso" para la sociedad. Ese contenido se despliega en diversos géneros, con el objetivo de transmitir mensajes, impartir conceptos y entretener, utilizando diversas técnicas, especialmente los dibujos animados, para captar en su audiencia a la niñez, especialmente. Estos dibujos son producidos en una diversidad de colores, tamaños y temas, muchas veces presentando contenido no aptos para su audiencia. Esta investigación propone, primero una clasificación de esos dibujos animados, según su contenido. Y en segunda instancia, comprender las preferencias de dibujos animados que los estudiantes de sexto grado de escuelas primarias oficiales urbanas, demuestran. La investigación focalizo dos casos de estudio, el de mayor y menor puntaje según las pruebas TERCE.

Palabras Claves: Dibujos animados; Pruebas TERCE; Educación; Pedagogía; Innovación educativa.

\footnotetext{
${ }^{1}$ Artículo elaborado a partir de la tesis de grado sustentada el 09 de junio 2017, por la estudiante y hoy Licenciada en Ciencias de la Comunicación Social por la Universidad Santa María La Antigua.

${ }^{2}$ Autora Principal. Licenciada en Ciencias de la Comunicación Social por la Universidad Católica Santa María La Antigua - USMA

${ }^{3}$ Autor Secundario. Investigador, Facultad de Ciencias Sociales, Universidad Santa María la Antigua - USMA.
} 
Invest. pens. crit. (ISSN 1812-3864)

Vol. 5, No. 2, mayo-agosto 2017

pp. 21-36

\section{Introducción}

El propósito de este estudio es medir y analizar la relación que tiene la exposición de dibujos animados, de los estudiantes en sexto grado de primaria, en escuelas oficiales en la República de Panamá, con el puntaje más alto y bajo a partir de los resultados de las pruebas internacionales TERCE. Luego de la selección de los casos de estudios, la investigación plantea dos interrogantes: una a nivel descriptivo y la otra en la dimensión correlacional. ¿Cuáles son las preferencias de los casos seleccionados sobre los tipos de contenido en los dibujos animados?, ¿Cuál es el grado de correlación entre el tipo de contenido de dibujos animados y el rendimiento académico en los casos seleccionados?

La televisión entre los demás medios resalta, por su capacidad de incidir en el público. En ocasiones, con valores socialmente aceptados y en otras, con una gran cantidad de antivalores y comportamientos no deseados en la conducta e interpretación social; es decir, juega un papel importante en la socialización del niño y niña.

Parte del contenido transmitido en televisión, son los populares dibujos animados, que consisten en figuras en movimiento compaginadas, con el fin de enviar mensajes claros y entretener a la audiencia más joven.

Aunque, existen criterios de selección, sobre el tipo de dibujos animados que deben observar y no deben observar los niños, de acuerdo con su edad. El mensaje es transmitido masivamente a todo el público, abriendo el compás a estímulos no apropiados para la edad del niño o la niña, influyéndolos de distintas maneras. En un mundo ideal, los adultos toman previsiones para condicionar qué tipo de contenido observan, sin embargo la realidad es otra.

La exposición prolongada a los medios de comunicación de masas, como lo es la televisión, ha creado una nueva especie. El homo sapiens, un ser caracterizado por la reflexión, por su capacidad para generar abstracciones, se está convirtiendo en un "homo videns", una criatura que mira pero que no piensa, que ve pero no entiende. A través de estas reflexiones, se pretende advertir del papel de los medios en nuestra sociedad y cómo el mal uso de los mismos; transforma a la sociedad en objetos maleables e ignorantes. (Sartori, 1995)

En un período que engloba los 5 últimos años, el sistema educativo panameño se ha enfrentado a una abismal cifra de reprobados. Cabe destacar, que el 2016 presentó un récord negativo con 48,864 alumnos que fracasaron el año, siendo está la suma de primaria, media y bachiller, de los cuales 13,984 eran estudiantes de primaria ${ }^{4}$.

Por otro lado según datos presentados en el "Atlas Social de Panamá: Acceso y uso de las tecnologías de información y comunicación”, presentado por el Ministerio de Economía y Finanzas (Censo, 2010) el 81.4\% de los hogares del país (incluyendo comarcas), cuentan o tienen acceso a un televisor, a cifras más exactas del mismo estudio reveló que en lo que se refiere a cantidad de televisores, Panamá tiene un total de 1.17 millones de televisores, lo que representan que en promedio cada hogar tenía más de uno. (Ministerio de Economía y Finanzas, 2010)

\footnotetext{
${ }^{4}$ Información publicada por el diario La Prensa el 12 de diciembre 2016, según datos del MEDUCA
} 
Invest. pens. crit. (ISSN 1812-3864)

Vol. 5, No. 2, mayo-agosto 2017

pp. 21-36

Esto quiere decir, que la mayoría de los niños y niñas de Panamá tienen acceso a los dibujos animados; sin contar que muchos utilizan dispositivos alternos como computadoras y teléfonos móviles.

El televisor, desde tiempos inmemorables, ha sido el medio de entretenimiento predilecto por la audiencia, lo que nos indica que dicho medio masivo tiene la capacidad de enviar un mensaje a una gran cantidad de la población y el propósito de dicho mensaje, ha sido objeto de debates por la sociedad, especialmente por la UNICEF.

La relación existente entre el consumo de televisión y el comportamiento de los niños, ha sido un tema de interés para las diferentes ciencias implicadas en el análisis e intervención sobre el comportamiento social colectivo, que se puede ver reflejado en el resultado académico de los infantes.

Aunque no existe una respuesta definitiva, se ha demostrado que la televisión posee efectos importantes sobre la conducta, pero subsisten interrogantes respecto a las condiciones bajo las cuales se presentan estos efectos. Dado que se trata de un fenómeno social global, la relación entre el medio televisivo y el comportamiento de las audiencias, trasciende el análisis e intervención de la conducta individual, de forma que se hace necesario emplear un marco más integrador e interdisciplinario para explicar y predecir los efectos.

Parte de este consumo excesivo de medios masivos, se da por parte de los niños, a través de los dibujos animados. Es importante recalcar que muchos de estos contenidos no se encuentran regulados y los niños no son supervisados por sus respectivos acudientes sobre la exposición a estas cómicas. Los dibujos animados tienen como objetivo, transmitir mensajes a su audiencia, por medio de dinámicas consideradas entretenidas, pero muchas veces con un alto contenido nocivo para el crecimiento del niño.

Estudios como el experimento del "Muñeco Bobo" (Bandura, 1961 y 1963), apuntan que el contenido de la televisión puede moldear la conducta y la percepción de los infantes en edades críticas del crecimiento, que aprenden a través de la observación y más tarde imitan. Debemos tomar en cuenta, a qué exponemos a los niños en formación, ya que estos preceptos anticuados podrán afectar su adecuado desarrollo.

Con el pasar de los años hemos transformado la televisión en una herramienta para la distracción de los jóvenes, abriéndole las puertas a un mar de información, con contenidos de otras culturas, ideas, creencias, actitudes, sin filtros que muchas veces escapan de su comprensión, pero que influyen en su conducta o pueden distorsionar sus valores.

Al demostrar un evidente gusto por las cómicas estos niños tienen la predisposición de querer emular las hazañas de sus héroes en dibujos animados. Este hecho puede resultar contraproducente para su comportamiento, porque algunos alegaban que el gusto de las cómicas se basaba en "la muerte de alguien" o "por qué se le pegaba a alguien", habían bombas, guerra o disparos, mentiras y burlas". (Rodríguez, 1995) 
Invest. pens. crit. (ISSN 1812-3864)

Vol. 5, No. 2, mayo-agosto 2017

pp. 21-36

El contenido de estos medios masivos de entretenimiento causa un efecto clásico, explicado en la "Teoría Social del Aprendizaje" y en el "Efecto Priming", que tratan sobre la repetición del contenido inadecuado a la vida real. La identificación que tienen estos escolares sobre determinados personajes, se manifiesta en la forma como actúan ante sus padres y maestros. (Rodríguez, 1995)

Otro elemento es que estos contenidos carecen de la supervisión adulta, tal como lo muestra la investigación sobre Violencia y Manipulación en la Programación Infantil de Televisión (Posada, 2007):

"Aun cuando los resultados de las encuestas a los familiares haya arrojado diversas críticas a la programación infantil de televisión, se desprende también de ellos, que los familiares no conocen la programación que ven los más pequeños, señalan más a los concursos o programas educativos, que las series y dibujos que en realidad les gustan. También responde, que siempre comentan con los niños la programación televisiva, sin embargo al no conocer bien los programas que los más pequeños ven, ni verlos con ellos, nos parece una respuesta engañosa”. (p.89)

Adicional los dibujos animados juegan un papel de importancia en la educación de los niños y la formación de espacios adecuados para el crecimiento óptimo (Patiño, 2011):

"Los niños y niñas, adquieren su identidad personal a medida que atraviesan por un proceso de socialización con su entorno, así también, los medios de comunicación juegan un papel importante en sus vidas, como los cuentos, las películas, etc., que transmiten información sobre los papeles o funciones de cada sexo y que son asimilados de forma inconsciente, hasta integrarlos como algo natural en su comportamiento". (p.190)

Estos efectos se pueden medir de manera palpable en edades críticas como la niñez temprana y media, debido a que el infante en cuestión, está en un proceso de desarrollo bastante susceptible y aprende a través de su entorno. Si bien, no se puede establecer una relación de causalidad, se sabe que están correlacionados. La televisión también puede provocar, con programas de contenido estereotipado, que los niños que tienen una vida diferente a los patrones establecidos, desarrollen un sentimiento de no pertenencia y a la vez se genere el rechazo de éstos por parte de los demás niños. (García, 2009)

Este contenido se ha posicionado como una de las posibles causas que desgastan a las nuevas generaciones, al no estar expuestas a hechos reales y valores ideales. Todos podemos comprobar la importancia que tienen los medios de comunicación en nuestra vida, y a la vez, lo prejudicial, que en ocasiones pueden llegar a ser para los niños, ya que en algunos casos, evidencia hechos de la vida sobre sexo, drogas, violencias, guerras, discriminaciones y alcohol, analizados sin ningún rigor científico ni ético. Hechos que perjudican a la mayoría, porque los valores reales, estilo de vida y manera de vivir de cada uno está "manipulado" por modelos, valores y tipos de comportamiento, algunos de los cuales están bastante fuera del alcance de la mayoría de los hombres, pero otros pueden ser imitados y ejercer influencia directa sobre nuestro comportamiento y sobre todo, sobre la conducta infantil". (Aldea, 2004) 
Invest. pens. crit. (ISSN 1812-3864)

Vol. 5, No. 2, mayo-agosto 2017

pp. 21-36

Es decir, que los medios pueden crear nuevas maneras de ver el mundo, basándose en antivalores y comportamientos no deseados, tomando en consideración que el niño en cuestión, es expuesto desde temprana edad a largas horas de contenido audiovisual, muchas veces no filtrado.

El niño, dado que tiene una mente plástica y muy receptora con todo lo que acontece a su alrededor, trata de asumir e interiorizar inconscientemente, todos los valores, roles, normas, actitudes y deseos que el mensaje televisivo le ofrece continuamente. La mayoría de los alumnos, tienen asumidas las actitudes negativas de los personajes televisivos que, cuando se sitúan delante de la pantalla del televisor, ven la violencia, peleas, agresividad, malas contestaciones, etc., como normales. La imagen que trasmiten los programas de televisión sobre personajes famosos, simpáticos, se transforma en deseable, admirable, imitable. Los niños los transforman en deseable, admirable, imitable. Los niños los transforman en sus favoritos, no distinguiendo la realidad de los mismos de la ficción, tampoco aprecian la bondad de sus conductas y comportamientos, sino que en función de la apreciación global del personaje favorito, sus acciones les sugieren imitación y grandeza o rechazo. (Perlado, 2003)

Los dibujos animados precedieron al cine mudo. Desde sus inicios, estos dibujos animados también conocidos como cómicas o caricaturas, eran enfocados en problemáticas sociales con contenido violento y direccionado para adultos. (Amurrio, 2015)

A partir de los años 60 , la popularidad del contenido animado, se trasladó a un nicho más joven convirtiéndose en la programación predilecta de los niños y adolescentes con el mismo contenido de sus inicios, representando una problemática en la familia al no poder supervisar el contenido al que sus hijos estaban expuestos.

Hanna - Barbera fue la encargada en los años 70 de acuñar la temática del "sueño americano", en la difusión masiva de sus dibujos animados, esta vez implementando recursos de sonidos "graciosos", para apaciguar el efecto de las escenas violentas.

El desarrollo de las cómicas en los años 80 dio un giro en el tipo de contenido que conocía para la época. Siguiendo la línea del "sueño americano", se inicia la producción de dibujos animados y largometrajes animados, basados en cuentos clásicos, introduciendo por primera vez, las figuras fijas conocidas en la literatura como los héroes, princesas y villanos que buscaban llegar al "y vivieron felices para siempre".

El "boom" de los dibujos animados dio un giro interesante, en la época de los 90's, con el inicio de la franquicia de princesas de Walt Disney World. Eran dibujos animados con estilo virginal que inspiran bondad y dulzura, lo que le brinda a los padres de familia, tranquilidad sobre la información a la que exponen a sus hijos.

Posteriormente, se vieron los dibujos animados con leve contenido violento o trazos de humor negro, no obstante eran camuflados con irrealismo como lo fue "Dragón Ball Z" o "Los Caballeros del Zodíaco", al igual que varios dibujos animados del género "ánime”.

No obstante, actualmente el contenido infantil de los dibujos animados está sobrecargado de información no apta para infantes, humor sexual, léxico inapropiado y diseños muy gráficos. 
Invest. pens. crit. (ISSN 1812-3864)

Vol. 5, No. 2, mayo-agosto 2017

pp. $21-36$

A partir de aquel momento, el contenido directo al que están expuestos los niños, se convirtió en un problema, ya que brindan modelos sociales no apropiados y antivalores.

Todo lo anterior contribuye a la preocupación que se tiene por el notorio desgaste de los valores familiares y las buenas costumbres. Es importante poder resolver estos conflictos reconociendo de una vez por todas, qué responsabilidad e influencia tiene el contenido de los dibujos animados con los cambios de conducta y el moldeo de una percepción nociva en los niños, para así lograr ajustes necesarios que permitan recuperar los valores.

La base a esta investigación, recae en la importancia de potenciar a los jóvenes a través de ambientes amenos y positivos, eliminando o sustrayendo estos estímulos que pueden estar causando interferencias con lo aprendido en el colegio o en el hogar. El objetivo primordial es brindar una serie de recomendaciones en base a los resultados, para guiar a los centros educativos, padres de familia y entidades que velan por el contenido, de cómo manejar la información masiva que se entrega mediante la programación.

Se quiere aclarar que esta investigación no pretende ofrecer lineamientos de censura del contenido, ya que iría en contra de la libertad de expresión, que es un pilar de la Comunicación Social, sino que desea brindar información sobre el efecto de los medios masivos en los más pequeños

\section{Algunas precisiones.}

A falta de una clasificación “oficial” o "estándar”, se propone una clasificación para esta investigación. Se le denomina tipología a una división, en este caso para los dibujos animados, tomando en consideración su imagen, objetivos, el desarrollo y los conceptos que transmiten.

Contenido Positivo: Se le considera a los dibujos animados que presentan conceptos reales, mensajes afirmativos, buenas costumbres y responden al conocimiento práctico y útil con relación a los estándares de la sociedad.

Contenido de Aprendizaje Académico: Enfocado en la difusión de mensajes que van acorde con los procesos de aprendizaje escolar y los planes de estudio universales como lo son: el abecedario, los números, palabras, problemas matemáticos y de lógica, entre otros.

Contenido de Aprendizaje Moral: Enfocado en la difusión de mensajes positivos a través del ejemplo. Dibujos animados que presentan buenas costumbres y enseñan valores, siguiendo modelos básicos de la convivencia en familia, el compañerismo, la amistad y el "buen actuar".

Contenido Negativo: Se le considera a los dibujos animados que perpetúen las malas costumbres, comportamientos inadecuados, mensajes ambiguos y van en contra de los estatutos sociales de la actualidad.

Contenido de Comportamiento Violento: Dibujos animados en los que se observan situaciones con altos contenidos de violencia, confrontaciones entre las personas, uso de armas y la presencia de sangre que perpetúan ejemplos negativos y erróneos. 
Contenido de Lenguaje y Conceptos Inadecuados: Dibujos animados en los que el mensaje y contenido están cargados de antivalores y malas costumbres, además de mostrar conceptos y acciones no aptas para los jóvenes. Utilizan lenguaje soez e inadecuado.

\section{Materiales y métodos.}

Esta investigación es tipo y alcance, descriptivo - correlacional. De enfoque cuantitativo - no experimental. Comparativo.

La investigación se realiza en dos centros educativos:

- centro educativo oficial urbano con el puntaje obtenido más alto de las pruebas TERCE.

- centro educativo oficial urbano con el puntaje obtenido más bajo de las pruebas TERCE.

- Variables de estudio: la primera variable nos permite seleccionar los casos, la segunda y tercera se busca establecer si hay o no asociación entre ambas.

\begin{tabular}{|c|c|c|c|}
\hline Variable & Definición Operacional & Dimensiones & Indicadores \\
\hline \multirow{4}{*}{$\begin{array}{l}\text { Pruebas } \\
\text { TERCE }^{5}\end{array}$} & \multirow{4}{*}{$\begin{array}{l}\text { Estudio que evalúa el } \\
\text { desempeño escolar en } \\
\text { sexto grado de escuela } \\
\text { primaria en áreas de } \\
\text { Matemáticas, Ciencias } \\
\text { Naturales y Lenguaje } \\
\text { (lectura y escritura) con el } \\
\text { objetivo de evaluar la } \\
\text { calidad de la educación en } \\
\text { la región. }\end{array}$} & Nivel I & $\begin{array}{l}\text { Inferior a } 686 \text { puntos en Matemáticas, } \\
\text { inferior a } 668 \text { puntos en Ciencias } \\
\text { Naturales y hasta } 611 \text { puntos en Lectura. }\end{array}$ \\
\hline & & Nivel II & $\begin{array}{l}\text { Entre } 687 \text { a } 788 \text { puntos en Matemáticas, } \\
669 \text { a } 781 \text { puntos en Ciencias Naturales y } \\
612 \text { a } 753 \text { puntos en Lectura. }\end{array}$ \\
\hline & & Nivel III & $\begin{array}{l}\text { Entre } 789 \text { a } 877 \text { puntos en Matemáticas, } \\
782 \text { a } 861 \text { puntos en Ciencias Naturales y } \\
754 \text { a } 809 \text { puntos en Lectura. }\end{array}$ \\
\hline & & Nivel IV & $\begin{array}{l}\text { Desde } 878 \text { puntos en Matemáticas, } 862 \\
\text { puntos o más en Ciencias Naturales y } 810 \\
\text { puntos o más en Lectura. }\end{array}$ \\
\hline $\begin{array}{c}\text { Dibujos } \\
\text { Animados }\end{array}$ & 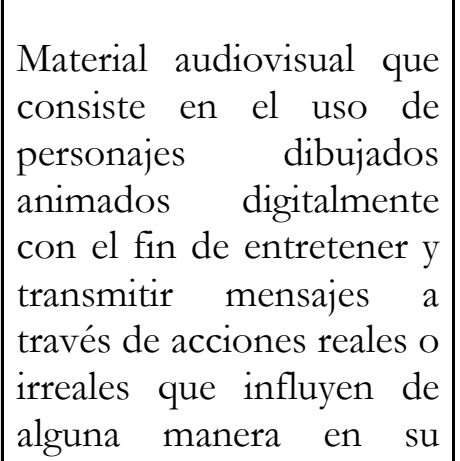 & $\begin{array}{l}\text { Contenido } \\
\text { Positivo }\end{array}$ & $\begin{array}{l}\text { Colores brillantes, buen léxico, palabras } \\
\text { positivas, mensajes de aliento, valores, } \\
\text { conceptos educativos, situaciones } \\
\text { sencillas, normas culturales, se premian las } \\
\text { buenas acciones, refuerzan relaciones con } \\
\text { amigos y familia, estimula los sentidos, } \\
\text { invita a la interacción con el ambiente, } \\
\text { casos de "problema - solución" y refuerzo } \\
\text { de acciones socialmente aceptadas. }\end{array}$ \\
\hline
\end{tabular}

${ }^{5}$ La definición operacional, dimensiones, indicadores ya están dados por el propio estudio Terce. 
Invest. pens. crit. (ISSN 1812-3864)

Vol. 5, No. 2, mayo-agosto 2017

pp. 21-36

\begin{tabular}{|l|l|l|l|}
\hline Variable & Definición Operacional & Dimensiones & \multicolumn{1}{|c|}{ Indicadores } \\
\hline $\begin{array}{l}\text { audiencia según su } \\
\text { contenido. }\end{array}$ & $\begin{array}{l}\text { Contenido } \\
\text { Negativo }\end{array}$ & $\begin{array}{l}\text { Colores opacos, mal uso de las palabras, } \\
\text { presencia de adjetivos calificativos } \\
\text { negativos, mensajes de derrota, valores } \\
\text { ambiguos, prácticas de anti valores, } \\
\text { rebelión ante las normas de la ley, se } \\
\text { perpetúan acciones negativas, refuerzan } \\
\text { malas conductas, confrontaciones de } \\
\text { violencia física y/o verbal, uso de armas, } \\
\text { presencia de sangre y lenguaje soez. }\end{array}$ \\
\hline
\end{tabular}

Población de estudio ${ }^{6}$ está formada por 118 estudiantes de sexto grado, compuesto por 61 niños y 57 niñas, en los cuales el 92\% tiene entre 11 y 12 años de edad.

Tabla 1 Edady sexo de los niños y niñas de las escuelas seleccionadas

\begin{tabular}{|c|c|c|c|c|}
\hline & & Niño & Niña & Total \\
\hline Edad & 11 años & 33 & 17 & 50 \\
\hline & 12 años & 28 & 30 & 58 \\
\hline & 13 años & 0 & 5 & 5 \\
\hline & 14 años & 0 & 1 & 1 \\
\hline Total & & 61 & 57 & 118 \\
\hline
\end{tabular}

El instrumento utilizado cuenta con diez (10) preguntas abiertas y cerradas, el mismo fue sometido a un proceso de validación por expertos y de igual manera se aplicó una prueba piloto en una escuela oficial urbana con alto y bajo puntaje similar a los de los casos seleccionados.

Pasos dados en la investigación:

El primer paso, fue descargar la base de datos de los resultados de las pruebas TERCE, que son de acceso público en el siguiente sitio de la UNESCO.

http://www.unesco.org/new/es/santiago/terce/databases/

El segundo paso, fue procesar la base de datos. Con el fin de seleccionar los casos de estudio, se utilizó la técnica de casos diversos para guiar la selección de casos. De acuerdo con (Seawright y Gerring, 2008), el objetivo principal de esta técnica es el de lograr la mayor variabilidad en las dimensiones relevantes, y tiene la mejor capacidad para argumentar representatividad en los estudios con muestras pequeñas.

\footnotetext{
${ }^{6}$ Se firmó acuerdo de confidencialidad con todos los actores (niños, niñas, maestros, maestras, padres y madres de familia, acudientes y director(a) de los centros educativos. Lo cual prohíbe mencionar los nombres de todos los que participaron.
} 
Invest. pens. crit. (ISSN 1812-3864)

Vol. 5, No. 2, mayo-agosto 2017

pp. $21-36$

El tercer paso fue utilizar un listado proporcionado por $\mathrm{MEDUCA}^{7}$ para verificar con precisión los casos escogidos, los nombres y determinar cuáles eran oficiales y particulares.

Luego de tener las escuelas agrupadas, podemos identificar los casos que interesan en esta investigación. Oficial urbana con puntaje más alto y oficial urbana con puntaje más bajo.

En ese sentido denominaremos como caso 1 (escuela oficial urbana que presenta mayor puntaje en las pruebas terce) y caso 2 (escuela oficial urbana que presenta menor puntaje en las pruebas terce)

Se aplicaron los Instrumentos en los casos seleccionados. En este punto queremos explicar que los niños solo enunciaban los tres dibujos animados que más observan, por ejemplo:

Mencione los dibujos animados que observa mayormente. (Pregunta $N^{\circ} 8$ del instrumento)

1.

2.

3.

Luego se analizaba el contenido de cada dibujo animado enunciado por los niños o niñas y de acuerdo a la operacionalización de la variable se codificaba la respuesta

En concordancia con los objetivos de la investigación, el procesamiento de la información extraída mediante el uso de la estadística, estableciendo las frecuencias de acuerdo con las respuestas, tablas cruzadas, comparando ambos casos.

\section{Resultados}

Los primeros elementos que aporta la información fue que las edades se concentran entre 11 y 12 años. Ahora se procede a analizar los resultados obtenidos. Al analizar la pregunta cerrada sobre la preferencia de ver dibujos animados, nos encontramos con los siguientes resultados.

Tabla 2 Preferencia de observar dibujos animados

\begin{tabular}{|l|r|r|r|r|r|r|}
\cline { 2 - 7 } \multicolumn{1}{c|}{} & \multicolumn{2}{c|}{ Válido } & \multicolumn{2}{c|}{ Casos Perdido } & \multicolumn{2}{c|}{ Total } \\
\cline { 2 - 7 } \multicolumn{1}{c|}{} & $\mathrm{N}$ & Porcentaje & $\mathrm{N}$ & Porcentaje & $\mathrm{N}$ & Porcentaje \\
\hline $\begin{array}{l}\text { Veo Dibujos } \\
\text { Animados }\end{array}$ & (si) 117 & $99.2 \%$ & 1 & $0.8 \%$ & 118 & $100.0 \%$ \\
\hline
\end{tabular}

Fuente: Datos derivados del presente estudio

Tenemos un caso perdido, un informante no respondió esta pregunta. Pero el primer elemento demuestra que todos los niños y niñas tienen una fuerte inclinación por ver dibujos animados, lo cual da sentido y coherencia seguir con el análisis de los datos obtenidos.

\footnotetext{
7 Se firmó un acuerdo de confidencialidad, el cual prohíbe divulgar tanto este listado como mencionar los nombres de los centros educativos que fueron seleccionados para esta investigación.
} 
Invest. pens. crit. (ISSN 1812-3864)

Vol. 5, No. 2, mayo-agosto 2017

pp. 21-36

La siguiente pregunta por analizar es: ¿qué consideras aprendes a través de los dibujos animados?

Tabla 3 Consideraciones de los niños sobre las temáticas de aprendizaje en los dibujos animados

\begin{tabular}{|c|c|c|c|c|}
\cline { 3 - 5 } \multicolumn{2}{c|}{} & Caso 1 & Caso 2 & Total \\
\hline \multirow{3}{*}{$\begin{array}{c}\text { Qué considero } \\
\text { aprendo de los } \\
\text { Dibujos } \\
\text { Animados }\end{array}$} & Valores & $60.7 \%$ & $72.4 \%$ & $63.6 \%$ \\
\cline { 2 - 5 } & Antivalores & $18.0 \%$ & $10.3 \%$ & $16.1 \%$ \\
\cline { 2 - 5 } & Nada & $21.3 \%$ & $17.2 \%$ & $20.3 \%$ \\
\hline
\end{tabular}

Fuente: Datos derivados del presente estudio

Los resultados de las tablas anteriores nos revelan información importante, que nos presentan la oportunidad de catapultar los métodos de enseñanza, utilizando como herramienta, los dibujos animados de contenido positivo.

La tabla $\mathrm{N}^{\circ} 4$ presenta los aspectos que más les gusta a los niños y niñas, en los dibujos animados. Este dato es importante, en función de que en primera instancia les gusta ver muchos dibujos animados, a su vez el 63.6\% considera que se aprenden valores; por lo tanto ahora es importante detectar que aspectos deben priorizarse en el contenido, si se pretende utilizar de forma pedagógica para la transmisión de conocimiento.

Tabla 4 Aspectos que priorizan los niños y niñas en los dibujos animados

\begin{tabular}{|l|c|c|c|c|}
\cline { 2 - 5 } \multicolumn{2}{c|}{} & $\begin{array}{c}\text { Caso } \\
1\end{array}$ & \begin{tabular}{c} 
Caso \\
\multicolumn{2}{c|}{}
\end{tabular} & Total \\
\hline \multirow{2}{*}{$\begin{array}{l}\text { Que es lo que } \\
\text { principalmente te } \\
\text { gusta de los } \\
\begin{array}{l}\text { Dibujos } \\
\text { Animados }\end{array}\end{array}$} & Caricaturas \\
\cline { 2 - 5 } & Fantasía & $23.6 \%$ & $14.8 \%$ & $21.6 \%$ \\
\cline { 2 - 5 } & Acción & $10.1 \%$ & $11.1 \%$ & $10.3 \%$ \\
\cline { 2 - 5 } & Da lo mismo & $13.5 \%$ & $11.1 \%$ & $12.9 \%$ \\
\hline \multicolumn{2}{|c|}{ Total } & $43.8 \%$ & $59.3 \%$ & $47.4 \%$ \\
\hline
\end{tabular}

Fuente: Datos derivados del presente estudio 
Invest. pens. crit. (ISSN 1812-3864)

Vol. 5, No. 2, mayo-agosto 2017

pp. $21-36$

Los datos que se presentan en la tabla $\mathrm{N}^{\circ} 5$, es coherente con los presentados en las tablas anteriores, al arrojarnos que el $83.2 \%$ de los niños y niñas observan entre 2 a 4 horas diarias dibujos animados. Como sociedad debemos aprovechar la atención que provocan los dibujos animado en los niños y niñas, lo irónico es que todos sabemos esto, empero no lo potenciamos para un proceso de socialización deseado.

\section{Tabla 5 Horas diarias que dedican a ver dibujos animados}

\begin{tabular}{|c|c|c|c|c|}
\hline & & Caso 1 & Caso 2 & Total \\
\hline \multirow{3}{*}{$\begin{array}{l}\text { Horas diarias } \\
\text { dedicadas a los } \\
\text { Dibujos Animados }\end{array}$} & $\begin{array}{l}\text { Menos de } 2 \text { horas al } \\
\text { día }\end{array}$ & $7.1 \%$ & $3.4 \%$ & $6.2 \%$ \\
\hline & $\begin{array}{l}\text { Entre } 2 \text { y } 4 \text { horas al } \\
\text { día }\end{array}$ & $84.5 \%$ & $79.3 \%$ & $83.2 \%$ \\
\hline & Más de 4 horas al día & $8.3 \%$ & $17.2 \%$ & $10.6 \%$ \\
\hline \multicolumn{2}{|c|}{ Total } & $100 \%$ & $100 \%$ & $100 \%$ \\
\hline
\end{tabular}

Fuente: Datos derivados del presente estudio

La siguiente variable evalúa la preferencia del niño y niña, en el tipo de contenido de dibujos animados. Lo presentado en la tabla $\mathrm{N}^{\circ} 6^{8}$, nos muestra que para el caso 2 los estudiantes tienen mayor preferencia por contenidos que implican comportamiento violento y lenguaje inadecuado. Este es uno de los puntos más importantes de este estudio, pues los resultados demostrarían si existe algún tipo de similitud o diferencia entre ambos casos con relación al tipo de contenido que se prefiere en el dibujo animado.

Tabla 6 Tipo de contenido en los dibujos animados que prefieren los estudiantes

\begin{tabular}{|c|c|c|c|c|}
\hline & & Caso 1 & Caso 2 & Total \\
\hline \multirow{2}{*}{$\begin{array}{l}\text { Tipo de } \\
\text { Contenido de } \\
\text { los Dibujos } \\
\text { Animados de } \\
\text { tu preferencia }\end{array}$} & $\begin{array}{l}\text { Aprendizaje } \\
\text { académico y/o moral }\end{array}$ & $46.1 \%$ & $24.1 \%$ & $40.7 \%$ \\
\hline & $\begin{array}{l}\text { Comportamiento } \\
\text { violento y lenguaje } \\
\text { inadecuado }\end{array}$ & $53.9 \%$ & $75.9 \%$ & $59.3 \%$ \\
\hline \multicolumn{2}{|c|}{ Total } & $100.0 \%$ & $100.0 \%$ & $100.0 \%$ \\
\hline
\end{tabular}

Fuente: Datos derivados del presente estudio

\footnotetext{
${ }^{8}$ Hay que explicar, que al estudiante no se le pide que indique el tipo de contenido de su preferencia. Ellos simplemente deben señalar los dibujos animados de su preferencia y luego los investigadores analizan el contenido de cada uno, codificándolos bajo esta variable dicotómica
} 
Invest. pens. crit. (ISSN 1812-3864)

Vol. 5, No. 2, mayo-agosto 2017

pp. 21-36

Si bien el caso 2, es la escuela que obtuvo menor puntaje en las pruebas Terce, en la tabla $\mathrm{N}^{\circ} 7$ aplicamos una prueba de chi-cuadrado, para corroborar si hay algún grado de asociación, entre ambas variables.

Tabla 7 Prueba de chi-cuadrado. Tipo de contenido de los dibujos animados y tipo de centro educativo.

\begin{tabular}{|c|c|c|c|c|c|}
\hline & Valor & df & $\begin{array}{l}\text { Significación } \\
\text { asintótica } \\
\text { (bilateral) }\end{array}$ & $\begin{array}{l}\text { Significación } \\
\text { exacta } \\
\text { (bilateral) }\end{array}$ & $\begin{array}{c}\text { Significación } \\
\text { exacta } \\
\text { (unilateral) }\end{array}$ \\
\hline $\begin{array}{l}\text { Chi-cuadrado de } \\
\text { Pearson }\end{array}$ & $4.359^{\mathrm{a}}$ & 1 & .037 & & \\
\hline $\begin{array}{l}\text { Corrección de } \\
\text { continuidad }^{\mathrm{b}}\end{array}$ & 3.498 & 1 & .061 & & \\
\hline $\begin{array}{l}\text { Razón de } \\
\text { verosimilitud }\end{array}$ & 4.573 & 1 & .032 & & \\
\hline $\begin{array}{l}\text { Prueba exacta de } \\
\text { Fisher }\end{array}$ & & & & .050 & .029 \\
\hline $\begin{array}{l}\text { Asociación lineal } \\
\text { por lineal }\end{array}$ & 4.322 & 1 & .038 & & \\
\hline $\mathrm{N}$ de casos válidos & 118 & & & & \\
\hline
\end{tabular}

Fuente: Datos derivados del presente estudio

a. 0 casillas $(0.0 \%)$ han esperado un recuento menor que 5 . El recuento mínimo esperado es 11.80 .

b. Sólo se ha calculado para una tabla 2x2

Los datos son consistentes con la tabla de contingencia, puesto que " $\mathrm{p}<0,05$ ", lo cual nos dice que existe algún grado de relación entre el tipo de contenido de los dibujos animados y tipo de centro educativo.

No obstante, es importante determinar la fuerza de relaciones entre ambas variables, por lo que se efectúan pruebas Phi y V de Cramer'. Los resultados que nos muestra la tabla $\mathrm{N}^{\circ}$ 8, nos confirman que el grado de correlación, no presenta la fuerza necesaria.

\footnotetext{
${ }^{9}$ Cramérs $\mathrm{V}=0$ : no hay relación entre $\mathrm{X}$ e $\mathrm{Y} /$ Cramérs $\mathrm{V}=1$ : hay una relación perfecta entre $\mathrm{X}$ e $\mathrm{Y}$ Cramérs $\mathrm{V}=0,6$ : hay una correlación relativamente intensa entre $X$ e $Y$; en ciencias sociales para poder señalar hay algún grado de correlación con fuerza importante por lo mínimo debe estar $\geq 0,3$
} 
Invest. pens. crit. (ISSN 1812-3864)

Vol. 5, No. 2, mayo-agosto 2017

pp. $21-36$

Tabla 8 Medidas simétricas para evaluar el grado de correlación entre ambas variables

\begin{tabular}{llr|r} 
& & Valor & $\begin{array}{c}\text { Significación } \\
\text { aproximada }\end{array}$ \\
\hline $\begin{array}{l}\text { Nominal por } \\
\text { Nominal }\end{array}$ & Phi & .192 & .037 \\
\cline { 2 - 4 } & \begin{tabular}{l} 
V de Cramer \\
\cline { 2 - 4 }
\end{tabular} & .192 & .037 \\
\hline $\begin{array}{l}\text { Coeficiente de } \\
\text { contingencia }\end{array}$ & .189 & .037 \\
\hline N de casos válidos & & 118 & \\
\hline
\end{tabular}

Fuente: Datos derivados del presente estudio

Estos resultados ocurren, ya que ambos casos están expuestos a los dibujos animados negativos tal y como se explicó anteriormente. Lo importante de esta variable tal y como se refleja en las tablas de contingencia, es la diferencia porcentual entre ambos casos. El caso 1 (colegio oficial urbano de alto rendimiento), muestra un balance entre la exposición de contenido positivo y negativo en los dibujos animados mientras que el caso 2, (colegio oficial urbano de bajo rendimiento), muestra una brecha elevada entre el valor porcentual de aquellos estudiantes que están expuestos mayormente a contenido positivo $(24.1 \%)$ y aquellos expuestos mayormente a contenido negativo $(75.9 \%)$.

\section{Conclusiones y recomendaciones.}

Los resultados apuntan a que existe un alto grado de interés por los dibujos animados de contenido violento y lenguaje soez, ya que los mismos tienen contenidos más atractivos que aquellos denominados educativos.

La investigación proporciona información valiosa que consideramos puede ayudar a tomar decisiones en materia de educación, para ser implementada por las autoridades rectoras de la educación en Panamá.

Los datos arrojan, que sin importar el estudiante, todos ven dibujos animados. Les gusta, dedican más de cuatro horas para verlos, involucran a los adultos.

Tal y como explica el Dr. Alan Rosenblatt en el documental "Life, Animated", sobre los beneficios de los dibujos animados en la educación de los niños autistas: "Son figuras dibujadas a mano con expresiones exageradas, con emociones exageradas. Es más fácil interpretar todo esto.”

Esta referencia abre el compás al uso de los dibujos animados, para explicar en los diversos niveles de primaria: conceptos complejos, introducción a valores y prácticas aceptables, sin perder las características atractivas, para los niños como los son la comedia y las situaciones dinámicas, así como el uso de material animado, meramente educativo con miras a los mismos objetivos. 
Invest. pens. crit. (ISSN 1812-3864)

Vol. 5, No. 2, mayo-agosto 2017

pp. 21-36

La era tecnológica nos incentiva a mejorar los procesos educativos, a través del uso del multimedia audiovisual que penetra de manera más atractiva que el clásico tablero y marcador.

A partir de lo anterior, se abre paso a una serie de recomendaciones destinadas principalmente, para el Ministerio de Educación (MEDUCA) de la República de Panamá:

- Utilizar dibujos animados en la educación primaria, como parte de las estrategias pedagógicas.

- Conformar un equipo de especialistas de la comunicación para que puedan construir dibujos animados, como herramientas educativas, para ser aplicados de forma transversal en todos los contenidos curriculares.

- Instalación de televisores en las aulas de clase para la implementación de herramientas tecnológicas y la proyección de material audiovisual animado, adecuado para el plan de estudio.

- Evaluación de películas y dibujos animados en búsqueda de contenido acorde a fines directos de la enseñanza primaria.

- Implementación de foros de discusión, con los estudiantes para evaluar su nivel de comprensión y medir los efectos de estos nuevos métodos en el aula de clase.

- Producción de contenido de dibujos animados netamente nacional, a través de universidades y profesionales de la comunicación, que vayan completamente en línea con el plan de estudio nacional, para explicar concepto científicos, nuestra historia, cultura, valores, tradiciones, los símbolos patrios, problemas sociales, entre otros.

- Educación para padres y madres, explicando los efectos de los dibujos animados en las edades de desarrollo del infante y cómo identificar el tipo de contenido al que su hijo está expuesto y cuánta influencia puede tener sobre su conducta.

- Los dibujos animados son una oportunidad educativa, una herramienta para hacerle frente a las debilidades del aprendizaje, como estrategia pedagógica, acorde a los contextos actuales y la aspiración de toda la sociedad de alcanzar la calidad educativa y una educación para todos.

\section{Agradecimientos.}

Queremos agradecer a la Secretaria Nacional de Ciencia y Tecnología (SENACYT), que en el marco del proyecto de investigación con código "ITE-15-007" se pudo acceder a los casos de estudio. 
Invest. pens. crit. (ISSN 1812-3864)

Vol. 5, No. 2, mayo-agosto 2017

pp. $21-36$

\section{Bibliografía}

\section{Artículos}

Aldea, S. (2004). La Influencia de la ‘Nueva Televisión’ en las Emociones y en la Educación de los Niños.

Hall, A. (2009) College Students' Motives for Using Social Network Sites and Their Relationships to Users' Personality Traits.

\section{Audiovisual}

Goldman, J. (Producer), \& Williams, R.R. (Director). (2016). Life, Animated [Documentary]. Country of origin: A\&E Indie Films.

\section{Libros}

American Psycological Association. (s.f). Putting the power of television to good use. de México.

Padua, J. (2000). Técnicas de investigación: aplicadas a las ciencias sociales, México: El Colegio

Panamá, Ministerio de Economía y Finanzas. (2010). Atlas Social de Panamá: Acceso y uso de las tecnologías de información y comunicación. Panamá, PA: Ministerio de Economía y Finanzas.

Sampieri, R., Collado, C. \& Lucio, M., (2014). Metodología de la investigación, México: McGraw - Hill.

Sartori, G. "Homo Videns", 1995, 2015, septiembre 2

\section{Tesis}

Amurrio, A. (2011). Los dibujos animados. Las mujeres en Disney. (Tesis de pregrado)

Cantillo, C. (2010). Perpetuación de Roles de Género en la Filmografía de Disney: de la Ingenua Blanca nieves a la Postmoderna Tiene (1937 - 2009) (Tesis de pregrado).

García, R. (2009). Influencia de las Caricaturas en los Niños de 4 a 6 Años de Edad, Que Habitan en el Municipio de Sola de Vega, Oaxaca. (Tesis pregrado)

Ospina, P., García, G., Chipana, D. \& Carrasco, M. (2014). Influencia de las Películas Animadas de Disney en los Niños de América Desde la Década de 1950 Hasta la Actualidad (Tesis de pregrado).

Patiño, K. (2011). Representación de la Mujer en las Princesas de los Filmes de Walt Disney (Tesis de pregrado).

Posada, A. (2015). Violencia y Manipulación en la Programación Infantil de Televisión (Tesis)

Rodríguez, R. (1995) "Los Programas de Dibujos Animados Televisados Un Medios de Entretenimiento o Violencia Para los Niños" (Tesis)

Sandoval, M. (2006). Los Efectos de la Televisión sobre el Comportamiento de las Audiencias Jóvenes desde la Perspectiva de la Convergencia y de las Prácticas Culturales. (Tesis)

\section{Web}

Cortez Bohigas, M.M. (n.d.)Definición de rendimiento escolar.

Delgado, J. (2014) .Efecto Bandwagon: Cuando sumarse a la masa es la alternativa.

Los Llanos, U. (2012). Conducta Social.

Orengo, J. (2013). Watson y el Conductismo | La guía de Psicología. 
Invest. pens. crit. (ISSN 1812-3864)

Vol. 5, No. 2, mayo-agosto 2017

pp. 21-36

Pedagogía, S. (2013). TEORIAS DEL APRENDIZAJE

Perlado, L. (2003). La Influencia de la Televisión en los Niños.

Real Academia Española. (s.f.). Diccionario de la lengua española (23.a ed.).

Regader, B. (n.d.). La Teoría del Desarrollo Psicosocial de Erikson.

UNESCO, ONG.(n.d.) "¿Qué es El TERCE?|Organización De La Naciones Unidas Para La Educación, La Ciencia y La Cultura”. unesco.org. 